\title{
Germanica
}

\section{Représentation du monde contemporain dans Strom de Hannah Dübgen et Wenn das Land still ist de Carsten Kluth}

Repräsentationen der zeitgenössischen Welt in Strom von Hannah Dübgen und Wenn das Land still ist von Carsten Kluth

Representations of Today's World in Strom by Hannah Dübgen and Wenn das Land still ist by Carsten Kluth

\section{Emmanuelle Aurenche-Beau}

\section{OpenEdition}

Journals

Édition électronique

URL : http://journals.openedition.org/germanica/2671

DOI : 10.4000/germanica.2671

ISSN : 2107-0784

Éditeur

Université de Lille

Édition imprimée

Date de publication : 30 décembre 2014

Pagination : 127-138

ISBN : 9782913857346

ISSN : 0984-2632

\section{Référence électronique}

Emmanuelle Aurenche-Beau, « Représentation du monde contemporain dans Strom de Hannah Dübgen et Wenn das Land still ist de Carsten Kluth », Germanica [En ligne], 55 | 2014, mis en ligne le 30 décembre 2016, consulté le 06 octobre 2020. URL : http://journals.openedition.org/germanica/2671 ; DOI : https://doi.org/10.4000/germanica.2671 


\title{
Représentation du monde contemporain dans Strom de Hannah Dübgen et Wenn das Land still ist de Carsten Kluth
}

\author{
Emmanuelle AURENCHE-BEAU \\ Université Lumière Lyon 2
}

Les deux romans sur lesquels nous nous appuierons pour donner un aperçu de la production des écrivains de la génération du tournant du millénaire sont deux romans parus en 2013 : Strom de Hannah Dübgen ${ }^{1}$ et Wenn das Land still ist de Carsten Kluth ${ }^{2}$. Les deux auteurs sont nés dans les années 1970 (Hannah Dübgen en 1977 et Carsten Kluth en 1972) et ont déjà derrière eux une riche expérience de vie et d'écriture. Hannah Dübgen a fait des études de littérature et de philosophie à Oxford et de musicologie à Berlin, elle a également passé une année à Paris $^{3}$; elle est dramaturge, auteurs de livrets d'opéra et d'une pièce de thêâtre Gegenlicht (2009) ${ }^{4}$. Carsten Kluth a fait des études de sciences politiques à Berlin et à New-York et a travaillé comme consultant,

1. - Deutsher Taschenbuch Verlag, München, 2013. Nous le citerons désormais en indiquant uniquement $\mathrm{S}$ suivi du numéro de la page.

2. - Piper Verlag, München, 2013. Nous le citerons désormais en indiquant uniquement $\mathrm{W}$ suivi du numéro de la page.

3. - http://www.goethe.de/kue/lit/aug/de12387690.htm consulté le 5-6-14.

4. - http://www.dtv.de/autoren/hannah_duebgen_16205.html consulté le 5-6-14. 
notamment auprès de la commission européenne ${ }^{5}$; il est aussi auteur de scénarios de films d'animation et a écrit un premier texte littéraire, Supernova, publié en ligne sur le site epubli6.

Dans les romans dont il sera question ici, ils s'attachent tous deux à donner une image du monde contemporain ${ }^{7}$. Leurs personnages sont des gens de leur génération vivant et se mouvant dans un monde d'échanges permanents. Ils sont présentés dans leur quotidien, face aux multiples défis de leur vie professionnelle et privée. Nous nous intéresserons tout d'abord à l'image que les deux ouvrages donnent de ce monde pour étudier ensuite de quelle manière ils font émerger une réflexion sur les valeurs et sur leur éventuelle remise en question. Nous nous interrogerons enfin sur la construction des deux romans en nous demandant dans quelle mesure elle témoigne d'un renouvellement des formes narratives.

Il est tout d'abord frappant de constater qu'aussi bien dans l'un que dans l'autre des deux romans, l'action se situe à l'échelle du monde, la « globalisation » apparaissant d'emblée comme constitutive de notre temps. Le personnage principal du roman de Carsten Kluth, par exemple, Harald Kronauer, est certes juge à Berlin (et l'action principale du roman se situe à Berlin), mais il est, en tant que juge, chargé d'affaires concernant des étrangers en situation irrégulière (l'une d'elle concerne un jeune homme originaire du Bengladesh) qui l'amènent à s'interroger sur les causes de l'émigration à partir de la notion de " réfugié climatique ». Cela le conduit petit à petit à découvrir certaines des solutions envisagées pour limiter ce type d'émigration, notamment celles que permettent des technologies nouvelles, en plein développement, et à effectuer un tour du monde des lieux les plus menacés par les phénomènes liés au réchauffement climatique (hausse du niveau de la mer, inondations...) pour mieux saisir la réalité et l'ampleur du problème. Sa femme Johanna, quant à elle, est galeriste et expose notamment les œuvres d'un artiste nigérian engagé, en disgrâce dans son pays car il dénonce précisément les risques liés aux manipulations du climat (W 74). Leur fille aînée, âgée de sept ans, d'autre part, est dans une école germano-chinoise plutôt élitiste dans laquelle ils l'ont inscrite après s'être laissés convaincre par un client de Johanna de l'intérêt de lui faire découvrir « la culture et la langue de la future grande puissance mondiale » (W 77). Et comme si tous ces éléments ne suffisaient pas

5. - http://www.piper.de/autoren/carsten-kluth-3656 consulté le 5-6-14.

6. - http://www.epubli.de/shop/buch/Supernova-Carsten-Kluth-Kluth/9532 consulté le 5-6-14.

7. - Hannah Dübgen indique dans des interviews qu'elle veut «parler du présent » (« von der Gegenwart erzählen » http://www.3sat.de/mediathek/?mode=play\&obj=38993, que son sujet est « le présent » (« die heutige Zeit »); Carsten Kluth, quant à lui, présente son roman comme un « roman de société » (« Gesellschaftsroman ») http://www.youtube. com/watch?v=XN3i7z26Vuc. 
à inscrire le roman et ses personnages dans un monde globalisé, les parents de Kronauer s'apprêtent à quitter leur maison berlinoise pour aller passer la moitié de l'année à Ténériffe !

Dans le roman de Hannah Dübgen, seuls trois des vingt chapitres se passent à Berlin et seul l'un des quatre personnages principaux du livre est allemand, Ada. Sa profession cependant (elle est documentariste) la conduit à travailler dans divers lieux du monde. Au moment où s'ouvre le roman, elle vient de tourner un film dans la bande de Gaza. Les autres personnages vivent également entre différents pays (aucun des personnages ne vit dans son pays d'origine). La pianiste japonaise qu'est Makiko vit à Paris et voyage dans le monde entier pour donner des concerts. L'ornithologue brésilien qu'est Luiz est installé en Israël, lieu de passage des oiseaux migrateurs qu'il étudie, et s'apprête à se rendre à un colloque aux États-Unis. L'homme d'affaires américain qu'est Jason enfin, parcourt le monde pour négocier des regroupements et des rachats d'entreprises.

Le monde dans lequel évoluent les personnages est donc un monde d'échanges constants dans tous les domaines. Dans le domaine culturel tout d'abord où ils sont à l'évidence facteur d'enrichissement. C'est ainsi que Makiko, formée par un maître japonais et influencée par l'art et la philosophie des jardins japonais (S 45) joue avec une sensibilité et une subtilité remarquables la musique romantique européenne, notamment les études de Chopin, ou que le fait d'être étrangère permet à Ada et à sa collègue Judith de poser un regard neuf sur une situation aussi complexe que celles des relations entre Israéliens et Palestiniens. C'est d'autre part grâce à ses contacts avec une galeriste allemande que le peintre nigérian du roman de Carsten Kluth peut faire connaître des œuvres qu'il ne peut exposer dans son pays, car elles représentent des hommes politiques ou des hommes d'affaires qui prennent selon lui des décisions « irresponsables » concernant notamment le domaine « hypersensible » qu'est celui du climat (W 74). Dans le domaine scientifique également, les échanges apparaissent comme fructueux, comme en témoigne l'exemple de Luiz.

Les effets de la globalisation dans le domaine économique, quant à eux, sont présentés dans les deux romans comme nettement plus problématiques : le profit et la loi du plus fort semblent y régner en maîtres, ainsi que l'illustre le personnage de Jason dans le roman de Hannah Dübgen : les opérations menées par l'homme d'affaires américain chargé de racheter des entreprises en difficulté pour les sauver de la faillite sont en réalité un moyen, pour le groupe pour lequel il travaille, d'acquérir peu à peu le monopole dans un secteur promis à un bel avenir

8. - On trouve en outre dans le roman diverses allusions au caractère multiculturel de la société allemande : l'éducatrice de la crèche où vont les fils de Kronauer est Ukrainienne et la colocataire de sa nièce est originaire d'Europe de l'est. 
avec l'épuisement des réserves de pétrole, celui des accumulateurs pour voitures électriques. Dans le livre de Carsten Kluth, Kronauer découvre, quant à lui, les énormes enjeux financiers liés au développement des technologies censées permettre de lutter contre les effets du changement climatique : soi-disant pour protéger les populations du tiers-monde de l'élévation du niveau de la mer et des inondations, en réalité pour protéger l'Occident de l'afflux de réfugiés climatiques, des entreprises européennes investissent dans des dispositifs tels que des digues ou des sortes de canons à sel capables de repousser les nuages porteurs de pluie (!). Sont clairement dénoncés ainsi les collusions entre hommes politiques et hommes d'affaires. Le roman montre en outre l'exploitation médiatique qui est faite de ces thématiques : au cours du voyage autour du monde qu'il effectue, comme on l'a vu, pour découvrir les lieux les plus menacés par le réchauffement climatique, Kronauer est en effet accompagné par une journaliste qui publie à leur retour toute une série de reportages. Et lui-même s'est laissé convaincre d'écrire avec cette journaliste un livre au titre éloquent, La Défense de l'Occident, se laissant ainsi manipuler par la fraction du parti conservateur proche des hommes d'affaires qui investissent dans ces nouvelles technologies (par laquelle il s'est laissé approcher à la suite de son jugement dans l'affaire du réfugié climatique originaire du Bengladesh) fraction qui s'oppose au moratoire que demande le secrétaire d'État au ministère de l'écologie qui appartient à un autre courant de ce même parti !

Un autre aspect du monde actuel abordé par les deux romans est celui des conséquences des modes de vie contemporains, notamment de la multiplication des déplacements et l'évolution des modes de communication, sur les relations sociales : en même temps que le développement des moyens de transport facilite les voyages à travers le monde, les nouvelles technologies de la communication permettent d'abolir les distances si bien que, pour reprendre la phrase placée en exergue du livre de Hannah Dübgen, « il n'y a plus ni proche ni lointain ». Dans l'un et l'autre des deux romans, les personnages communiquent constamment par téléphone portable ou par messagerie électronique. Kronauer semble même n'avoir jamais été plus au courant des détails de la vie quotidienne de sa famille que lorsqu'il effectue son voyage autour du monde et qu'il reçoit des messages de sa femme ou de sa mère qui garde les enfants. Dans le roman de Hannah Dübgen, la distance géographique et la rareté de leurs rencontres ne semblent pas gêner Makiko et Gerald $^{9}$ ou Jason et Leyla ${ }^{10}$ - Jason souligne même explicitement qu'ils

9. - Makiko vit à Paris, Gerald à Londres.

10. - Jason vit là où le conduisent ses missions professionnelles, Leyla vit à Londres. 
sont proches même s'ils sont éloignés géographiquement ${ }^{11}$. Le paradoxe de ces relations à distance étant peut-être que la proximité physique ou la perspective d'une plus grande proximité physique devient difficile, comme l'illustrent quelques situations empruntées aux deux romans. Lorsqu'elle découvre qu'elle est enceinte, Makiko, en effet, est paniquée, elle apprécie l'intensité des brefs moments passés avec Gerald et ne semble guère avoir envie d'une relation quotidienne ou plus régulière ${ }^{12}$. Il est frappant de constater aussi que Kronauer souligne que les meilleurs moments de la relation avec sa femme sont ceux où ils se voient peu car ils sont chacun occupés de leur côté (c'est le cas notamment pendant la campagne de promotion de son livre qui l'amène à faire une tournée de lectures dans toute l'Allemagne ${ }^{13}$ ). Quant aux moments où ils sont ensemble, ils sont présentés de manière quelque peu caricaturale comme des moments où ils n'arrivent pas à se parler. Leurs conversations semblent se limiter à des aspects logistiques, sans que les questions de fond ne soient abordées, comme c'est le cas par exemple à propos du livre de Kronauer qui, à cause des nombreux voyages qu'il implique, se réduit à une «question d'organisation » sans que son contenu ne soit entre eux un sujet de discussion (W 11). On peut citer comme autre exemple la scène (W 280-283) où Kronauer annonce à Johanna qu'il a adhéré au parti conservateur et où cette dernière ne manifeste aucune réaction - il est vrai que le moment n'est peut-être pas très bien choisi : ils sont dans l'escalier roulant d'un centre commercial où Johanna veut acheter des chaussures à leur fille ! Lorsqu'ils sont chez eux, ils sont souvent décrits comme étant chacun occupé à répondre à ses appels téléphoniques ou à ses SMS si bien qu'il faudrait presque modifier la seconde partie de la phrase qui se trouve en exergue de Strom et dire non pas : «il n'y a plus que proche ou étranger », mais « il n'y a plus que proche et étranger »... La situation de Luiz et de sa femme est d'ailleurs un peu semblable, leurs vies semblent devenues parallèles et ils semblent ne plus partager grand-chose : alors que Luiz ne supporte plus

11. - « Dass Leyla nicht hier war, war nicht das Problem, die knapp neuntausend Kilometer zwischen ihnen empfand er nicht als quälend, im Gegenteil, er mochte sogar das Gefühl, sich geographisch weit entfernt und gleichzeitig so nah zu wissen, nur einen Anruf, eine Nachricht, eine elektrische Schwingung voneinander entfernt. » (S, 39-40).

12. - « Sie waren die einzigen Zeugen ihrer im Verborgenen lebenden Geschichte. Einer Geschichte ohne gemeinsames Zähneputzen, ohne Ringe, ohne Besitz. Einer Geschichte der reinen Lust, des kostbaren Augenblicks. », S 147.

13. - «Das Erscheinen des Buches hatte die Machtverhältnisse in ihrer Ehe ein wenig verschoben. Und es kam Kronauer vor, als hätte die Spannung nachgelassen. », W 271; «Im Juli, im August und im September absolvierte Kronauer eine Reihe von Lesungen [...] Auch Johanna hatte viel zu tun, und es gab Wochen in diesem Sommer, in denen sie nur miteinander telefonierten, um sich abzustimmen. Doch es kam Kronauer vor, als akzeptierte Johanna sein neues Leben. », W 277. 
la vie en Israël et l'obsession de la sécurité qui règne dans ce pays ( $\mathrm{S}$ 23-24), sa femme veut encore croire à une paix possible entre Israéliens et Palestiniens et s'engage dans une association qui tente de favoriser le dialogue.

Si les deux romans abordent donc ainsi indirectement les effets des nouvelles technologies de la communication sur les relations privées et familiales en les présentant de manière plutôt critique, le roman de Hannah Dübgen en fait néanmoins apparaître d'autres aspects, notamment les possibilités de communication avec l'extérieur qu'elles offrent dans un pays coupé du monde comme la bande de Gaza avec l'exemple des jeunes Palestiniennes rencontrées par Ada qui lancent sur internet un manifeste pour un Gaza libre qui recueille de nombreuses réactions (S 209).

En arrière-plan de cette description quasi phénoménologique de notre monde contemporain, les deux romans proposent aussi une réflexion sur la place des valeurs traditionnelles dans la société contemporaine. La famille, ou plus exactement la question des différentes conceptions possibles de la famille, semble y tenir une place importante. Le roman de Carsten Kluth met ainsi face à face, de façon là aussi peut-être un peu simpliste, le modèle contemporain, celui de Kronauer et de sa femme Johanna, et celui de la génération de leurs parents. Aux remarques récurrentes de sa mère qui ne cesse de répéter que Johanna travaille trop ${ }^{14}$, Kronauer oppose une nouvelle conception du couple où les deux conjoints sont engagées dans une vie professionnelle et se partagent les tâches du ménage et de l'éducation des enfants, ce qui ne va pas sans difficultés, sans tensions et sans fatigue, comme le montre le roman! Force est de constater en tout cas que tous ses engagements n'empêchent pas Kronauer d'être un père très présent auprès de ses enfants : alors que Johanna a très rapidement repris son travail à la galerie, c'est lui qui a pris un congé parental à la naissance du troisième, car il ne pouvait envisager de le laisser si petit à la garde d'une tierce personne ; il se montre également profondément bouleversé quand il apprend que sa fille aînée a été insultée à l'école à la suite du jugement qu'il a rendu dans l'affaire de l'émigré en situation irrégulière et il ne se remet pas d'avoir oublié ses deux fils dans la voiture pendant la conférence de presse à laquelle il avait promis d'être présent - cet oubli est même peut-être pour lui comme un signal qui lui fait comprendre qu'il est allé trop loin et qui le fait réfléchir à ses priorités : carrière politique, notoriété ou famille ?15

14. - On apprend qu'elle travaille parfois jusque tard dans la nuit à la galerie (W 9) ou qu'elle a recommencé à travailler très rapidement après la naissance de son troisième enfant (W 38).

15. - À la fin du roman, il renonce à sa carrière politique au nom de la préservation de sa vie familiale (W 380). 
Alors que les deux romans sont par ailleurs très différents l'un de l'autre, il est frappant de constater que c'est aussi autour de la question de la famille que se développe dans Strom un questionnement sur les valeurs. Le roman propose ainsi différentes situations opposant une conception «traditionnelle » et une conception « moderne » du couple et de la famille qui peuvent susciter la réflexion du lecteur. On comprend par exemple que Makiko a quitté le Japon pour fuir une société extrêmement conservatrice représentée dans le roman par sa mère Fumiko dont le comportement est avant tout dicté par des normes extérieures et par un souci constant de sauvegarder les apparences (S 111) et par sa cousine Mai qui a accepté un poste bien en dessous de ses qualifications afin d'assumer ainsi publiquement la faute de son père qui, coupable de malversations financières, a lâchement fui à l'étranger et ainsi déshonoré la famille dont il s'agit désormais de restaurer le « visage ${ }^{16}$. Elle entend, quant à elle, vivre de manière plus libre et plus individualiste, en prenant en compte ses propres désirs et en étant moins dépendante des normes sociales si importantes pour sa mère - elle revendique une relation de couple fondée sur « les sentiments sans les conventions » ${ }^{17}-$, et sa grossesse imprévue vient la placer devant un choix cornélien : pour quoi optera-t-elle, pour sa brillante carrière internationale de pianiste soliste et pour sa relation libre avec Gerald ou pour son enfant ou trouvera-t-elle un moyen de concilier tout cela ? Le personnage de Luiz est lui aussi confronté à différentes conceptions du couple, de la famille et de la société : opposé à celle d'une certaine frange de la population israélienne tentée par le repli identitaire et la fermeture sur soi, il tente de vivre avec sa femme d'une manière plus ouverte, au risque de voir son couple voler en éclats car chacun finit par vivre sa propre vie indépendamment de celle de son conjoint. Jason, quant à lui, qui parcourt le monde et a, comme on l'a vu, une relation surtout à distance avec une femme mariée, semble aspirer à une plus grande stabilité et à une relation plus « classique $»^{18}$. Le personnage d'Ada enfin ouvre peut-être sur une autre forme de lien que le lien familial, celui de l'amitié qui l'unit à sa coréalisatrice Judith qu'elle accompagne jusque dans les derniers instants du cancer qui l'emporte prématurément.

Notons aussi que Kronauer est également soucieux de l'avenir de sa nièce en échec scolaire qu'il tente d'aider à passer le bac en cours du soir.

16. - « Fumiko hatte ihren Mann gebeten, Mai in seiner Firma unterzubrigen, und das nicht, um ihrer Nichte einen Gefallen zu tun, sondern weil es für sie das einzig Denkbare war, dass ein Kind öffentlich für das Vergehen seines Vaters einsteht. », S 112.

17. - « [...] für Fumiko gab es nur das Aussen. Gerald und sie dagegen waren ein reines Innen, das Gefühl ohne die Konvention. », S 147.

18. - Il la presse de prendre une décision et de choisir entre son mari et lui (S 83-86). 
Outre les questions liées à la famille, on trouve également dans les deux romans des éléments de réflexion autour d'une autre valeur fondamentale, le respect dû à la nature. Le livre de Carsten Kluth pose la question de savoir jusqu'où l'homme peut modifier la création - si le défunt mari de la maîtresse de Kronauer était convaincu, sur la base de la parole biblique selon laquelle l'homme doit « dominer ${ }^{19}$ le monde créé par Dieu, qu'il est légitime que l'homme acquière la maitrise des phénomènes climatiques et météorologiques, on trouve aussi dans le roman des allusions à des phénomènes de protestation contre le «progrès », comme le combat de la jeune activiste qui détruit les voitures (W 44). Dans le roman de Hannah Dübgen, la nature est présente très discrètement à travers le titre d'un morceau joué par Makiko, « Flut » (Flot), une composition de son maître japonais qu'elle joue pour la première fois à Venise et qui évoque le phénomène des catastrophes naturelles (on pense notamment aux tsunamis). Et dans son discours, le directeur de la biennale qui l'a invitée met précisément en garde contre l'hybris qui consiste à se mettre au-dessus de la création et réclame davantage d'humilité devant la nature (S 49).

Alors que cette dimension semble absente dans Wenn das Land still ist, Strom évoque en outre une autre valeur traditionnelle, celle de la fierté nationale, comme le montre l'exemple de la solidarité dont font preuve les entreprises japonaises pour sauver Kazedo, entreprise traditionnelle, fleuron de l'industrie nippone, dont elles ne conçoivent pas, au grand dam de Jason, qu'elle puisse être rachetée par un groupe américain ${ }^{20}$.

La lecture des deux romans montre donc que certaines valeurs traditionnelles restent présentes au cœur d'un monde en plein bouleversement. Même si le sens de l'appartenance à une nation, le respect de la nature ou la pérennité des couples y sont parfois mis à mal, il est frappant de constater que la fidélité à l'histoire d'un pays, le respect de la création divine ou la famille par exemple restent des repères forts.

Nous voudrions pour conclure nous interroger sur la construction des deux romans et nous demander dans quelle mesure les choix narratifs opérés par les deux auteurs permettent de donner une image du monde qui nous entoure, notamment en ce qui concerne la place du sujet ou le rapport à l'espace et au temps, et dans quelle mesure ils présentent des aspects innovants.

19. - « Remplissez la terre et dominez-la. Soumettez les poissons de la mer, les oiseaux du ciel et toute bête qui remue sur la terre! », Genèse 1, 28, Traduction œcuménique de la Bible, Éditions du Cerf, 1977.

20. - Son directeur explique ainsi à Jason : « [...] wir haben es im Fall Kazedo mit einem japanischen Traditionskonzern zu tun. Da hängt mehr dran als nur Zahlen $[\ldots] », \mathrm{~S} 129$. 
Pour décrire notre monde, Carsten Kluth fait, comme on l'a vu, le choix de se concentrer sur un personnage principal, le juge Kronauer, qui semble être pour lui comme un archétype du représentant de la classe moyenne dans une société occidentale avec les différents défis auxquels il se doit de répondre : vie professionnelle, vie familiale, relation de couple, sans compter, dans son cas, son engagement politique. Afin de donner une idée concrète et précise de sa vie quotidienne, il le décrit en quatre chapitres sur une période de 24 heures, de son réveil à $3 \mathrm{~h} 54$ à son coucher à 4 heures du matin la nuit suivante, un jour d'août d'une année qui n'est pas datée ${ }^{21}$. Cette " journée particulière », particulièrement riche en événements de toutes sortes permet de le montrer dans ses différentes relations : ses relations à sa femme (on assiste à leurs échanges sur leurs contraintes professionnelles respectives et sur la répartition des tâches liées aux enfants ou au ménage); ses relations à ses enfants qu'il emmène à l'école ou à la crèche tout en préparant une interview à la radio; sa relation à sa maîtresse qui ne cesse de lui téléphoner; ses relations à ses collègues du tribunal, à sa secrétaire ; ses relations à ses amis politiques qui sollicitent sa présence à une conférence de presse au cours de laquelle le secrétaire d'État au ministère de l'écologie doit annoncer sa démission ; ses relations à sa nièce à qui il demande de garder ses enfants pendant ce temps; ses relations à ses parents qui les ont invités à dîner pour fêter leur départ de Berlin et leur installation à Ténériffe. Mais le roman ne se contente pas de cette trame narrative chronologique et la fait alterner avec des chapitres en forme de flash-backs qui décrivent trois moments-clés de la vie de Kronauer au cours des dix-huit mois précédents : le jugement à propos du réfugié climatique à l'origine de son engagement politique, le voyage hautement médiatisé qu'il entreprend quelques mois plus tard pour se rendre compte de visu de la réalité du changement climatique ; sa sélection comme candidat à la députation onze mois avant la journée d'août déjà évoquée. Kronauer apparaît ainsi comme le centre d'un riche réseau relationnel, mais le roman le présente aussi comme un être extrêmement naïf et manipulable. Il se laisse notamment largement instrumentaliser par sa maîtresse Martina et par les autres membres du parti conservateur qui utilisent son jugement à propos du réfugié climatique afin de favoriser les hommes d'affaires qui ont investi dans les nouvelles technologies d'intervention sur les phénomènes climatiques avec qui ils sont liés et afin de mettre fin à la carrière politique d'Ebené, le secrétaire d'État au ministère de l'écologie, qui réclame un moratoire sur ces technologies. À la demande de Martina qui lui dicte quasiment les réponses à donner, il accepte, comme on l'a vu, de répondre à l'interview d'une

21. - Les chapitres en question portent les titres suivants : 03.54-15.14, 15.14$16.02,16.02-16.26,16.26-4.00$. 
journaliste ; son jugement acquiert ainsi une dimension médiatique et il se retrouve pris dans un engrenage qui l'amène par la suite à participer à un talk show télévisé au cours duquel il s'engage malgré lui à participer à ce fameux voyage sur les lieux les plus menacés par le réchauffement climatique. Le livre qu'il publie à la suite de ce voyage et dont le titre accrocheur, La Défense de l'Occident, lui est imposé est ensuite largement médiatisé, les tournées de lectures qu'il effectue le font connaître, la conséquence logique de tout cela est que, quand Martina lui demande d'adhérer à son parti alors que ses idées politiques ne le portent pas du tout de ce côté-là, il ne peut faire autrement que d'accepter. Il est ensuite sollicité pour se présenter à des élections partielles afin de remplacer un député qui a dû démissionner pour raisons de santé et est à deux doigts de devenir, toujours malgré lui, secrétaire d'État en remplacement d'Ebené, à la suite de ce qui s'avèrera être une campagne de diffamation menée par Martina et ses amis politiques. C'est seulement in extremis qu'il se rend compte qu'il ne maîtrise plus rien de sa vie et qu'il finit par prendre conscience qu'il a été manipulé. Kronauer apparaît ainsi comme le représentant de l'homme contemporain qui perd son « centre » et se laisse ballotter par les circonstances ${ }^{22}$. Le roman tout entier, en effet, par sa forme de chronique (heure par heure dans les chapitres 1,3,5,7 qui relatent une journée de la vie de Kronauer, la fameuse journée du 12 août ; jour par jour dans le chapitre 4 qui décrit son voyage autour du monde, mois par mois dans les chapitres 2 et 6 qui résument son ascension politique) donne au lecteur l'impression que les événements s'enchaînent à un rythme effréné qui ne permet ni pause, ni respiration, ni réflexion. Peut-être Kronauer aspire-t-il finalement, tout simplement, à la fin du roman à une certaine forme de calme et de paix, comme le suggère le titre du livre ?

Les choix narratifs à l'œuvre dans Strom semblent eux aussi répondre au projet de donner une image fidèle de notre monde, mais ils y répondent d'une manière différente. Au lieu de se centrer sur un personnage central, Hannah Dübgen en effet fait le choix, comme on l'a vu, d'entrecroiser les destins de quatre personnages qui vivent dans quatre pays différents et qui représentent quatre domaines d'activité différents. Ils sont comme quatre échantillons représentatifs (?) de la vie dans notre monde globalisé. Comme s'il était pour elle impossible, pour rendre compte de son caractère éclaté, de se centrer sur un person-

22. - Cette idée apparaît de manière incidente dans le roman : au cours du trajet en voiture qui le conduit chez ses parents, il s'interroge sur ce qui donne sens à sa vie et repense au sermon qu'il a entendu lors du mariage d'une cousine, en particulier à cette phrase : « ton cœur est là où est ce que tu as de plus précieux » et il se rend compte qu'il ne sait plus où il en est parce qu'il ne sait plus ce qui est le plus important pour lui (W, 339-340). 
nage et de raconter sa vie de manière linéaire, on a dans son roman de brefs fragments discontinus de la vie des différents personnages (les chapitres consacrés aux différents personnages alternent). Le dernier chapitre du roman cependant réunit les quatre personnages autour d'un concert donné par Makiko à Jérusalem : Luiz et sa femme s'y rendent après la manifestation pour la paix organisée à l'occasion de la visite du président américain ; Jason, en voyage d'affaires en Israël, y est présent aussi ; Ada s'y retrouve après avoir enterré les cendres de son amie Judith sur les lieux où elles ont tourné leur documentaire. S'agit-il pour Hannah Dübgen d'offrir une nouvelle variante de sa réflexion autour de la dialectique du proche et du lointain qui lui semble caractéristique de notre monde et dont elle dit dans une interview qu'elle est l'idée d'origine du livre ?23 Ici les quatre personnages principaux sont en effet à la fois lointains, étrangers les uns aux autres puisqu'ils ne se connaissent pas et proches physiquement puisque réunis dans un même lieu (Ada et Jason sont même assis côte à côte). Est-ce à dire que l'art peut être un moyen de réunir les hommes, à l'image de la musique de Makiko, à l'image aussi de l'illustration de la couverture du livre, un tableau de Gerhard Richter qui représente des lignes parallèles de couleurs différentes qui, réunies, forment un ensemble, un tout qui est davantage que la juxtaposition de chacune de ses parties ou encore à l'image du roman lui-même qui tisse à l'insu des personnages un réseau de liens entre eux ? C'est ainsi en effet, pour ne donner que quelques exemples, que Jason a, au Japon, la cousine de Makiko comme secrétaire; Rachel, la femme de Luiz, quant à elle, écoute un disque de Makiko; Luiz doit se rendre à un congrès aux États-Unis, alors que Jason vient en Israël pour rencontrer les dirigeants d'une entreprise qui investit dans des accumulateurs solaires dont le beau-frère de Luiz est un fervent partisan; à Tel Aviv, Ada loge chez une jeune femme qui n'est autre que la maîtresse de Luiz. Tout se passe donc comme si les histoires respectives des quatre personnages principaux (eux-mêmes connectés, comme on l'a vu, à d'autres personnes à travers le monde grâce au réseau électrique des multiples connexions téléphoniques ou électroniques) étaient comme les affluents d'un grand fleuve qui pourrait être le fleuve de la vie, faisant du titre du roman, Strom, le concentré du livre tout entier.

L'un et l'autre des deux romans semblent donc nous donner une image assez réaliste du monde contemporain dans ses différents aspects : globalisation, multiplication des déplacements et des échanges dans tous les domaines, conséquences de ces changements pour l'individu, désir de répondre à la fois aux défis de la vie professionnelle et aux exigences de la vie privée et familiale, difficulté à trouver le temps de la

23. - «Das war ein Ur-Gedanke », http://www.unicum.de/studienzeit/leben/ buecher/unter-strom-autorin-hannah-duebgen-im-interview. 
pause et de la réflexion... Même si chacun des deux auteurs semble ainsi faire une place à des phénomènes pouvant sembler caractéristiques de notre temps, comme l'éclatement spatial et temporel, la dialectique du proche et du lointain, du centre et du réseau, leurs choix narratifs nous semblent cependant rester assez traditionnels (narrateur omniscient, narration chronologique) et on peut regretter qu'ils ne se risquent pas à davantage d'expérimentation littéraire. 